\section{TIM3 suppresses antitumour DCs}

T cell membrane protein 3 (TIM3) is an immune checkpoint receptor that suppresses the activation of Thelper 1 $\left(T_{H} 1\right)$ cells on engagement with ligand. It is also expressed by dendritic cells (DCs), which are part of the innate immune system that respond to tumour-derived stress-related factors. The function of TIM3 in innate antitumour immune responses is unclear.

Chiba and colleagues confirmed that TIM3 is expressed by tumour-associated DCs isolated from patients with cancer. TIM3 was also expressed at high levels by DCs and macrophages - as well as by $\mathrm{CD} 8^{+} \mathrm{T}$ cells - that were associated with implanted tumours in syngeneic mice. Consistent with innate immune responses being a first line of defence, the authors found that the levels of TIM3 expression were higher, and were induced earlier, on tumour-associated DCs than on tumour-infiltrating $\mathrm{CD} 8^{+} \mathrm{T}$ cells. TIM 3 induction on immature bone marrow-derived DCs co-cultured with mouse tumour cell lines was synergistically prevented by the blockade of vascular endothelial growth factor receptor 2 (VEGFR2), interleukin-10 (IL-10) and arginase 1 , which are associated with immunosuppressive signalling.
What is the role of TIM3 on tumour-associated DCs? The authors found that $\mathrm{TIM}^{+}{ }^{+} \mathrm{DC}$ s produced lower levels of pro-inflammatory cytokines when treated with Toll-like receptor 3 (TLR3), TLR7 and TLR9 agonists, such as nucleic acids. By contrast, bone marrow-derived DCs isolated from $\mathrm{Tim}^{-1-}$ mice that were treated with various immunostimulatory nucleic acids showed increased activity of the TLR signalling mediators interferonregulatory factor 3 (IRF3) and nuclear factor- $\mathrm{\kappa} \mathrm{B}(\mathrm{NF}-\mathrm{\kappa} \mathrm{B})$ and had a higher cytokine response than TIM3+ DCs. Similarly, mRNA levels and secretion of the cytokines interferon- $\beta 1$ (IFN $\beta 1$ ) and IL-12 were reduced in tumour-associated TIM $3^{\text {hi }}$ DCs isolated from tumour-bearing mice or from patients with cancer. Treatment of tumour-bearing mice with TIM3-specific monoclonal antibodies (mAbs) that block ligand binding increased the secretion of IFN $\beta 1$ and IL- 12 in response to nucleic acids. Therefore, TIM3 suppresses the activation of tumour-associated DCs in response to immunostimulatory nucleic acids.

To determine the effect of TIM3 expression on DC antitumour immune responses, the authors treated mice bearing syngeneic B16-F10 melanomas with TIM3-specific mAbs and immunostimulatory nucleic acids, which substantially reduced tumour growth. TIM3-specific mAbs and immunostimulatory nucleic acids had the same antitumour effects in CD8 ${ }^{+} \mathrm{T}$ cell-deficient mice, indicating that TIM 3 expression by
DCs (and not T cells) confers reduced responsiveness to immunostimulatory nucleic acids. Furthermore, the authors found that TIM3 interacts with high mobility group protein B1 (HMGB1), which forms complexes with immunostimulatory nucleic acids to allow their endocytosis and, in turn, the activation of innate immune signalling. Further investigation revealed that TIM3 prevented HMGB1-mediated endocytosis of immunostimulatory nucleic acids in DCs.

The release of nucleic acids from dying tumour cells is thought to elicit antitumour innate immune responses, which might contribute to the efficacy of chemotherapy. Therefore, TIM3 expression may impair such therapeutic responses. Indeed, the authors found that blockade of TIM3 increased the expression of cytokine transcripts in $\mathrm{TIM}^{+}$DCs that were co-cultured with dying tumour cells that had been treated with cisplatin, and this was suppressed by the neutralization of HMGB1. Moreover, treating mice with TIM3 mAbs plus cisplatin significantly reduced the size of MC 38 syngeneic tumours in vivo, and the absence of $\mathrm{TIM}^{+}$DCs improved the inhibition of tumour growth by cisplatin in vivo.

Therefore, blockade of TIM3 could be used in combination with immunostimulatory nucleic acids and/or cytotoxic chemotherapy to improve therapeutic responses.

Gemma K. Alderton, Senior Editor, Nature Reviews Cancer

This article is modified from the original in Nature Rev. Cancer (doi:10.1038/nrc3349).

ORIGINAL RESEARCH PAPER Chiba, S. et al. Tumor-infiltrating DCs suppress nucleic acidmediated innate immune responses through interactions between the receptor TIM-3 and the alarmin HMGB1. Nature Immunol. 29 Jul 2012 (doi:10.1038/ni.2376) 